\title{
ROSA Analyser: An automatized approach to analyse processes of ROSA *
}

\author{
Raúl Pardo and Fernando L. Pelayo \\ Dept. de Sistemas Informáticos \\ E. Superior de Ingeniería Informática de Albacete \\ Universidad de Castilla - La Mancha \\ Campus Universitario. 02071-Albacete, Spain \\ raulpardo@dsi.uclm.es, FernandoL.Pelayo@uclm.es
}

\begin{abstract}
In this work we present the first version of ROSA Analyser, a tool designed to get closer to a fully automatic process of analysing the behaviour of a system specified as a process of the Markovian Process Algebra ROSA. In this first development stage, ROSA Analyser is able to generate the Labelled Transition System, according to ROSA Operational Semantics.

ROSA Analyser performance starts with the Syntactic Analysis so generating a layered structure, suitable to then, apply the Operational Semantics Transition rules in the easier way. ROSA Analyser is able to recognize some states identities deeper than the Syntactic ones. This is the very first step in the way to reduce the size of the LTS and then to avoid the state explosion problem, so making this task more tractable.

For the sake of better illustrating the usefulness of ROSA Analyser, a case study is also provided within this work.
\end{abstract}

\section{Introduction}

Formal methods are more used as Computer Science becomes a more mature science; this happens due to the fact that formal methods provide software designers with a way to guarantee high security and reliability levels for their computer science systems, and what is more, formal methods would allow to find software errors in the earliest stage of the software development life cycle so making it significatively cheaper. The main problem is that for systems with a size or complexity level, the analysis could be difficult to be developed, mainly because of two reasons, the first comes from the size of the graphical model usually generated, the second is because the most times these analyses are made by hand; we begin with the latter so presenting a tool to apply automatically the operational semantics of the Process Algebra, ROSA. In the next future we will continue dealing with the problem of the size of the LTS generated by ROSA.

During the past 20 years many efforts have been done in order to develop support tools for all kinds of formal methods. For instance for timed automata UPPAAL tool [1] gives to designers a good environment to design using timed automata, as well as, it supports analyzing skills. In the Petri Nets field, TINA is a frequently used tool [2]. Regarding Process Algebras, several tools have been developed also, the most known could be PEPA Workbench [3] which was developed based on PEPA process algebra [4]. The main problem of process algebra based tools is to deal with the state explosion arisen in the Labelled Transition System (LTS), because of this, some of the process algebra research lines are searching the way to reduce the size of the LTS to be generated, and so to avoid this problem as much as possible [8].

${ }^{*}$ This work has been partially supported by projects TIN2009-14312-C02-02 \& CGL2010-20787-C02-02

C. Andrés and L. Llana (Eds.): 2nd Workshop on Formal Methods in the Development of Software 2012 (WS-FMDS 2012).

EPTCS 86, 2012, pp. 25-32 doi 10.4204/EPTCS.86.4 (c) Raúl Pardo \& Fernando L. Pelayo

This work is licensed under the Creative Commons Attribution License. 
In this paper we present a tool based on ROSA process algebra [5], so this tool has to deal with non-deterministic, probabilistic and temporal behaviours for a given process, which means that it has to provide a proper syntax for capturing all these features. Moreover, an easy and clear user interface has been designed for it which has been not very common in previously developed tools.

In its first development stage ROSA Analyser is able to build the LTS from a given ROSA process, by means of applying all of the possible operational Semantics rules which must be used for a given input process. In addition, for the sake of avoiding the state explosion problem, we are working on defining an heuristic which allows to reach from each state/process the most probably path to a given (final) state/process, by changing its exponential computational cost to a linear computational cost. Therefore, it involves an initial attempt to solve this problem and as a consequence of this, get a more practical way to analyse concurrent and real time systems by means of process algebra based tools.

The paper is structured as follows, section 2 provides a detailed description of ROSA Analyser, pointing out the type of used data structures and the analyzing process which makes possible the LTS building; in section 3 a case of use of it is presented, specifically a cognitive memorizing process has been analysed. To finish with, section 4 states both conclusions and future work.

\section{ROSA Analyser tool}

ROSA Analyser has been developed in JAVA, this choice was taken due to the facilities to handle with complex data structures, by which is characterized this programming language. In addition, ROSA Analyser is implemented over GPL v2 license, in order to allow everybody the possibility to work on the defined data structures. The source code and the executable version can be found in http://kenai. com/projects/semantictreerosa.

In order to show the way in which ROSA Analyser works, we will describe all of the main parts involved in the LTS generator. Below paragraphs detail the data structures over which ROSA Analyser works, then the Semantics analyser, containing the main work load, is executed so producing a kind of layered structure appropriate to be used by the latter process which builds the whole LTS form the original syntax expression of the input ROSA process.

\subsection{Data structures}

The theoretical process expressions with which ROSA Analyser works are not optimized for the analysis in which the tool has to be involved, due to this fact, the data are needed to be parsed in order to reach a better structure for their analysis processes. Specifically, in this section we show how the tool handle the data structures required for the Syntax and Semantics analysis.

\subsubsection{Syntax Tree}

As a result of the syntax analysis a binary tree is built with which can be made an easy and efficient Semantics analysis. In this Syntax analysis the higher syntactical priority the elements have, the closer to the top they are located, as previously said this structure is optimized to check the conditions of the upper side of the rules and to do the necessary modifications to the process which is being analysed.

In order to show in a clear way this data structure an example of the syntax tree of the ROSA process $<a, 0.3>.0 \|_{\{a, c\}}<b, \infty>.0$ can be seen in figure 1 .

In this example, we can see the root node corresponded with the highest priority element of the process, also this structure allows the semantics analysis to get directly the element which chooses the 


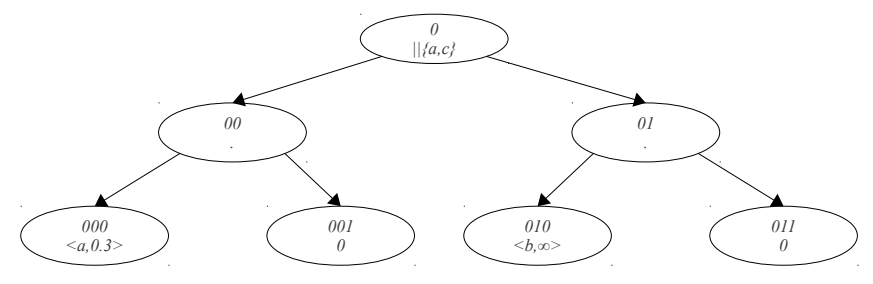

Figure 1: Syntax Tree Example

rule to be applied, and by means of defined methods also make possible change the Syntax tree so as to apply the selected rule. Detailed description about the building of the Syntrax tree will be provided.

\subsubsection{Semantics Tree}

Once we have defined a proper structure for the process' syntax we are ready to show the Semantics tree data structure which represents the LTS for a ROSA process. The LTS is built with nodes which are themselves ROSA processes, therefore in each Semantics tree node will appear a Syntax tree, this can show the high complexity by which is characterized this data structure. Also, unlike the Syntax tree, the transitions between nodes must get more information than those of the Syntax tree which further increase the amount of data to be handle.

In this case the defined tree structure is chosen with the aim to support the natural structure of the Semantics tree, which is a n-ario tree, unlike the previous data structure which was chosen looking for making easier the Semantics analysis. A Semantics tree example can be seen in figure 2.

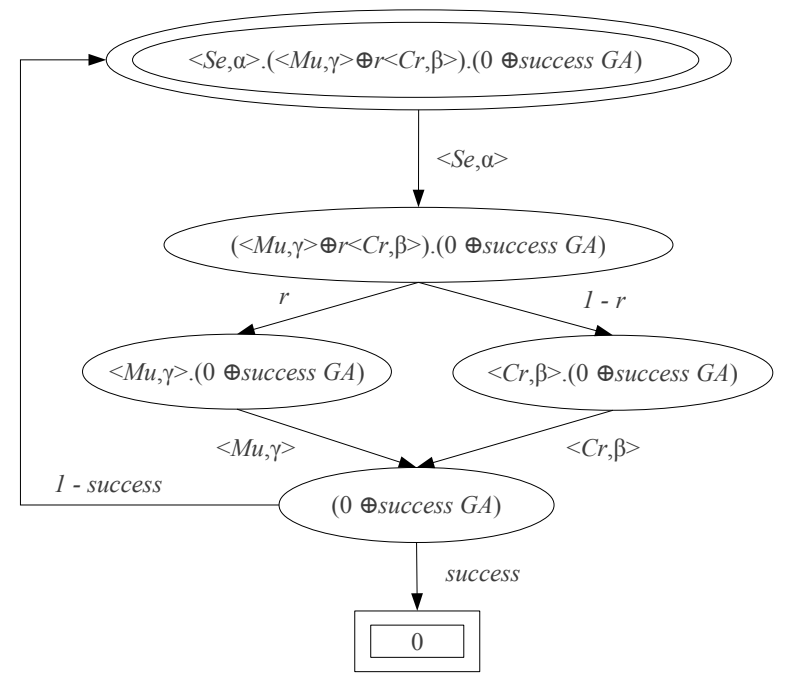

Figure 2: Semantics Tree Example

It is important to point out that this data structure also has several methods in which some functionalities which help to build the semantics tree have been implemented, such as joining new nodes to the 
Semantics tree, where in addition, a search for Syntax equivalent nodes is made everytime that a tentative "new" node is generated.

\subsection{Syntax Analysis}

\subsubsection{Tool input Syntax}

The Syntax analysis consists of the part of the tool which takes as input the plain expression of a ROSA process and returns the Syntax tree associated with that process. It was necessary to define some constrains for the input syntax because the ASCII characters do not provide the wide variety of elements by which is characterized ROSA Syntax. In the following table 1 are shown the equivalences between ROSA Syntax and the tool input Syntax:

\begin{tabular}{|c|c|}
\hline ROSA Syntax & Tool Syntax \\
\hline$a$ & $a$ \\
$<a, \alpha>$ & $<a, \alpha>$ \\
$a . P$ & $a . P$ \\
$<a, \alpha>. P$ & $<a, \alpha>. P$ \\
$P$ & $P$ \\
$P ; Q$ & $P ; Q$ \\
$P \oplus Q$ & $P-Q$ \\
$P+Q$ & $P+Q$ \\
$P \oplus_{r} Q$ & $P *\{r\} Q$ \\
$P \|_{A} Q$ & $P \| A Q$ \\
\hline
\end{tabular}

Table 1: Equivalence between ROSA and tool Syntax

Moreover, it is important to point out that the parameter $\alpha$ capturing the temporal behaviour of actions must be a real number, and the parameter $r$, a probability, must be a real number within the interval $[0,1]$. The set $A$ represents the synchronization set for a parallel operator, so that this set is $A=\{a, b, c, \ldots\}$.

\subsubsection{Syntax tree building}

Once the ROSA Analyser input method has been defined we are ready to describe the Syntax analysis, the first point to take into account is the priority of the operators, by default the priority is defined as follows:

1. Sequential processes operator.

2. Parallel operator.

3. External, internal and probabilistic choices.

4. Prefix.

5. Actions and process variables.

These priorities can be changed according to our needs by means of using parenthesis, according to their common behaviour, becoming a more flexible Syntax for the input method. 
Taking into account the priorities above defined, the Syntax analysis is mainly a recursive method in which the expression element with the highest priority must be found, then a node with the element is created or added to the Syntax tree, once reached this point if the element is an operator, the method splits the expression into two parts taking as split point the position in which was located the operator found, then the same method is called using both expressions, and the new elements found in these recursive calls are added to the Syntax tree which is being built, on the other hand, if not, i.e. if the element with the highest priority is an action or a process variable this element is added as a node to the Syntax tree but the recursive calls finish.

\subsection{Semantics Analysis}

Through this Semantics analysis is built the LTS for a ROSA process, the complexity of this analysis is very big since it has to determine which for all of the ROSA Semantics rules can must be used in order to show all of the possible behaviours that a given process can perform.

Firstly, a LTS constituted with a unique Semantics node which contains the Syntax tree build for the input process is created, this node will be the root node in the Semantics tree. In the next step it has to be determined which rule for all of the ROSA operational Semantics rules can be applied. As it can be seen in ROSA operational Semantics there are three rule sets (non-deterministic, probabilistic and action transition rules). Then the first checking in which ROSA Analysis is involved is to determine whether the process is non-deterministic by means of deterministic stability function, if the process is non-deterministic it will be checked which of the non-deterministic transition rules must be used, that will be checked by means of the root node in the Syntax tree.

On the other hand if the process is deterministically stable, the tool must determine trough the action function if the process can be evolved by probabilistic or action transition rules. Once the transition rules set has been chosen, ROSA Analyser determines, by means of the upper condition, which rule must be applied. In order to show a clearly way of this process, figure 3 shows its activity diagram.

Once all these nodes have been created, they must be joined to the LTS. As it is well-known the state explosion problem arises when the LTS is being built, but this tool, in order to reach a lighter solution for this problem, is able to detect Syntax identities between nodes in order to avoid a node to appear more than once. When the new nodes have been added to the LTS, again all leaf nodes will be analysed, in order to search more nodes that could be generated. This loop is repeated until two consecutive iterations do not provide any new node, this will mean that the LTS is completed.

\section{Analysing a memorizing process}

In order to show the usefulness of this tool, in this section we describe the way in which a memorizing process is analysed. This process was specified using ROSA in [7].

Now we are going to describe the input process and the equivalences with the original specification. However, to get a clearer description we roughly describe each process involved and its corresponding ROSA specification.

\section{Encoding process}

This is the process with which the memorizing process starts, firstly elements identification is computed, once this initial identification is complete then at the same time it tries to identify objects and relations with previous data. The ROSA specification for this process can be seen in (1): 


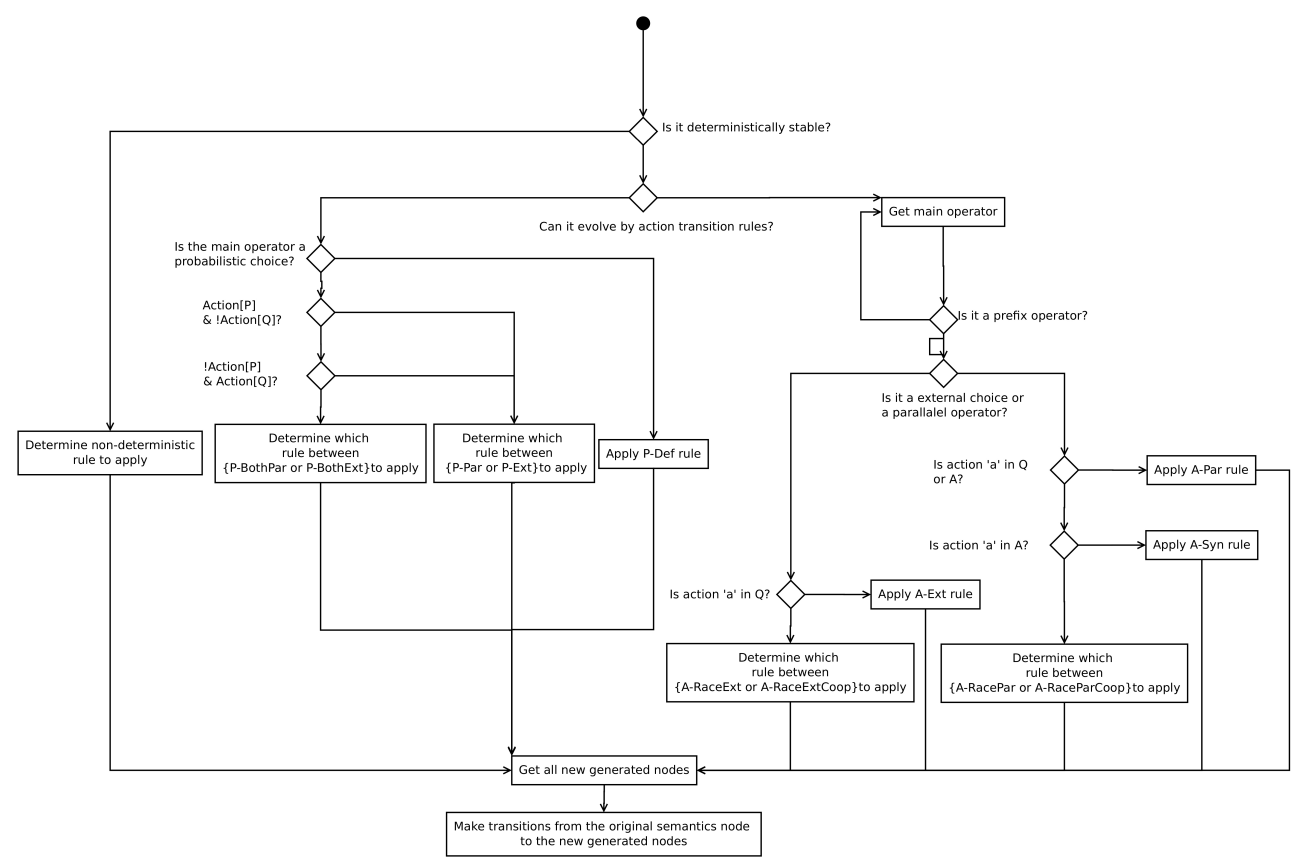

Figure 3: Activity diagram for Semantics nodes analysis process

Encoding $\equiv<$ Perception, $\beta>.<$ Start_Identification, $\infty>.\left(<\right.$ Find_Attributes, $\alpha_{1}>.<$ stm, $\infty>\|_{\{\text {stm }, \text { abm }\}}$

$$
\left.\|\|_{\{\text {stm,abm }\}}<\text { Identify_Object, } \alpha_{2}>.<\text { stm, } \infty>\|_{\{\text {stm,abm }\}}<\text { Find_Relations, } \alpha_{3}>.<\text { stm, } \infty>\right)
$$

The specification used as input for the tool is:

$$
E \equiv<a, 0.1>. b .(<c, 0.2>. f\|\{f, i\}<d, 0.3>. f\|\{f, i\}<e, 0.4>. f)
$$

\section{Consolidation process}

The memorizing process specified has three stages for memory storage: Sensory store, Short term memory and Long term memory. The first two kinds of memory aren't definitive, i.e. not consolidated yet, and the information perceived is stored several seconds there, but when the information is longer stored, we are consolidating this information. Because of this, once the encoding process has finished with a certain amount of probability (according to parameter $r$ in full memorizing process specification) the perceived information can be stored in long term memory or what is the same, it is definitively consolidated. The ROSA specification for this process is:

$$
\text { Consolidation } \left.\left.\equiv<\text { Soon_Rehearsal_or_Finding_Cues, } \alpha_{4}\right\rangle .<\mathrm{ltm}, \infty\right\rangle .<a b m, \gamma>
$$

In the same way that the previous process was used, the specification for ROSA Analyser is:

$$
C \equiv<g, 0.5>. h .<i, 0.6>
$$




\section{Retrieval process}

Retrieval process represents the way in which the memorizing process access to information which has been previously stored, ROSA specification for this process is:

$$
\text { Retrieval } \equiv<\text { Request }, \infty>.<a b m, \sigma>
$$

Therefore, as ROSA Analyser input we will use:

$$
R \equiv j \cdot<i, 0.7>
$$

\section{Losing process}

With a certain probability it is possible to lose the perceived information, this event can happen in the case the information does not reach the long term memory. Obviously, this process only represents a single action execution, so in the specification there is only one action involved:

$$
\text { Losing } \equiv<\text { Fading, } \alpha_{5}>
$$

For tool Syntax we take:

$$
L \equiv<k, 0.8>
$$

\section{LTS for the memorizing process}

Finally, joining all of the defined process the expression associated for the memorizing process is:

$$
\text { Mem } \equiv \text { Encoding } ;\left(\text { Consolidation } \oplus_{0.25} \text { Losing }\right) \|_{\{a b m\}} \text { Retrieval }
$$

And for ROSA Analyser this process must be written as follows:

$$
M \equiv E ;(C *\{0.25\} L) \|\{i\} R
$$

Once the Semantics analysis has been performed, ROSA Analyser provides two outputs, one in which the nodes and transitions can be textually seen, and other graphical output that by means of graphviz the LTS, can be exported to pdf, jpg, svg and eps. In url http://raulpardo.files.wordpress.com/ $2012 / 05 / \mathrm{m}$.jpg we can see the latter.

As a matter of proof of usefulness of ROSA Analyser, this LTS has two distinguished states, one red coloured representing a deadlock state, and a green coloured state representing a success.

\section{Conclusions and future work}

To sump up we can say that a tool to automatize the LTS building process of a ROSA process has been developed. This tool parses the process Syntax expression into a layered data structure quite suitable to better develop the Semantics analysis, such Operational Semantics provides the rules among which a proper subset of them, for each process should be applied. As a matter of fact, we have shown a real example by which the practical use of ROSA Analyser can be seen. In addition, it is important to mention 
that this tool provides two kinds of outputs, a textual output in which all nodes and transition can be seen; and a graphical view in which the Semantics tree is drawn in a portable format.

Although we reach a more practical use of ROSA process algebra, the state explosion problem has not been resolved yet. At this moment, ROSA Analyser is able to "see" more identities of processes than the Syntactical one so reaching a little reduction in the states number. However, as we previously stated some first steps in this line have been done, specifically, a metric over the set of ROSA processes and a sort of normal form has been defined in [6], through this distance and normal forms can be defined several heuristics which provide the tentative more promising branch from any state to a given final one, so decreasing the number of states and making this task more practically tractable.

\section{References}

[1] Johan Bengtsson, Kim Larsen, Fredrik Larsson, Paul Pettersson \& Wang Yi (1996): UPPAAL - a tool suite for automatic verification of real-time systems. In Rajeev Alur, Thomas Henzinger \& Eduardo Sontag, editors: Hybrid Systems III, Lecture Notes in Computer Science 1066, Springer Berlin / Heidelberg, pp. $232-243$. Available at http://dx.doi.org/10.1007/BFb0020949. 10.1007/BFb0020949.

[2] B. Berthomieu *, P.-O. Ribet \& F. Vernadat (2004): The tool TINA - Construction of abstract state spaces for petri nets and time petri nets. International Journal of Production Research 42(14), pp. 2741-2756, doi $10.1080 / 00207540412331312688$.

[3] S. Gilmore \& J. Hillston (1994): The PEPA Workbench: A Tool to Support a Process Algebra-based Approach to Performance Modelling. In: Proceedings of the Seventh International Conference on Modelling Techniques and Tools for Computer Performance Evaluation, Lecture Notes in Computer Science 794, Springer-Verlag, Vienna, pp. 353-368, doi:10.1007/3-540-58021-2_20.

[4] Jane Hillston (1996): A Compositional Approach to Performance Modelling. Cambridge University Press, doi:10.1017/CBO9780511569951.

[5] F. L. Pelayo (2004): Application of formal methods to performance evaluation. Ph.D. thesis, Universidad de Castilla - La Mancha.

[6] Fernando L. Pelayo, Fernando Cuartero \& Diego Cazorla (2011): Looking for a cheaper ROSA. In: Proceedings of the 11th international conference on Artificial neural networks conference on Advances in computational intelligence - Volume Part II, IWANN'11, Springer-Verlag, Berlin, Heidelberg, pp. 380-387. Available at http: //dl . acm . org/citation . cfm?id=2023332.2023387.

[7] Maria L. Pelayo, Fernando L. Pelayo, Fernando Cuartero, Valentin Valero, Gregorio Diaz \& Elena Nieto (2007): Does ROSA provide a good view of the Memorizing Process? In: Proceedings of the 6th IEEE International Conference on Cognitive Informatics, COGINF '07, IEEE Computer Society, Washington, DC, USA, pp. 273-283, doi:10.1109/COGINF.2007.4341900

[8] A. Stefanek, R.A. Hayden \& J.T. Bradley (2011): GPA - A Tool for Fluid Scalability Analysis of Massively Parallel Systems. In: Quantitative Evaluation of Systems (QEST), 2011 Eighth International Conference on, pp. 147-148, doi:10.1109/QEST.2011.26 